LA--10953-MS

DE87 007713

\title{
The f Electron Collapse Revisited
}

\author{
B. I. Bennett
}

\section{DISCLAIMER}

This report was prepared as an account of work sponsored by an agency of the United States Government. Neither the United States Government nor any agency thereof, nor any of their employees, makes any warranty, express or implied, or assumes any legal liahility o.' responsibility for the accuracy, completeness, or usefulness of any information, apparatus, product, or process disclosed, or represents that its use would not infringe privately owned rights. Reference herein to any specific commercial product, process, or service by trade name, trademark, manufacturer, or otherwise does not necessarily constitute or imply its endorsement, recommendation, or favoring by the United States Government or any agency thereof. The views and opinions of authors expressed herein do not necessarily state or reflect those of the United States Government or any agency thereof. 
THE $f$ ELECTRON COLLAPSE REVISITED

by

B. I. Bennett

\section{ABSTRACT}

A reexamination of the collapse of $4 \mathrm{f}$ and $5 \mathrm{f}$ electrons in the lanthanide and actinide series is presented. The calculations show the well-known collapse of the $f$ electron density at the thresholds of these serjes along with an $\mathrm{f}^{2}$ collapse between thorium and protactinium. The collapse is sensitive to the choice of model for the exchange-correlation potential. and the behavicr of the potential at large radius.

\section{Introduction}

The "collapse" of the $f$ electronic wavefunctions at the threshold of the lanthanide and actinide series has been studied by a variety of researchers. A summary of their results is presented in Ref. 1 (Table 8-8). The qualitative explanation for this phenomenon is given in terms of the competition between the coulomb-like atomic poteritial of the atom and the angular momentum barrier potential, which have opposite signs. The combination can produce a "double-well" effective potential.

If the resulting eigensolution has the appropriate energy, it is possible to have an appreciable probability of finding the electron at large radius. As the atomic number is increased, the coulomb-like attractive potential increases, causing an increased probability of finding the electron at smaller radius. Since the atomic number increases in discrete steps, this contraction of the eigensolution exhibits a somewhat abrupt collapse.

The purpose of this study is to examine the details of the phenomenon for the $4 \mathrm{f}$ and $5 \mathrm{f}$ electrons and investigate the properties of the $5 \mathrm{t}$ eigensolutions as the atomic number increases from 90 (Th) to 91 (Pa). 
The Mode1

The calculations are carried out using the self-consistent field program DAVID, ${ }^{2}$ which obtains one-electron solutions of the Dirac equation with a local density approximation for the exchange-correlation potential. Sensitivity studies are done for two exchange-correlation models: $\mathrm{Kohn}^{-\mathrm{Sham}^{3}}$ and Gunnarsson-Lundqvist. 4

The model potential includes a so-called Latter tail correction. ${ }^{5}$ This tail correction restricts the large radius potential to be $1 / r$ (in Hartrees). This behavior is physicaliy plausible for examining loosely bound and excited states for isolated atoms.

Since the solutions to the Dirac equation have major and minor components, the resulis are displayed in terms of the radial probability, $R_{\alpha}(r)=$ $4 \pi r^{2}\left|\psi_{\alpha}(r)\right|^{2}$, rather than the wavefunctions. The Kohn-Sham exchange-correlation potential is used to examine the phenomena of $f$ electron collapse, however, a different choice can dramaiically effect the results for the atoms at the threshold.

The $4 f$ Collapse

Figure 1 shows the radial probabilities of the $4 f_{5 / 2}$ wavefunctions for the excitej states of barium and lanthanum, and the ground state of cerium. The $f$ electron in barium, as has been observed by others, has its largest probability at a large atomic iadius (nearly 20 Bohr units). The addition of a $6 \mathrm{~s}$ electron, with the attendant increase in atomic number to give the excited configuration of lanthanum, shifts the maximum of the probability to less than 1 Bohr unit. There remains a small shoulder in the probability curve at the larger radius. Finally, adding another electron to a $5 \mathrm{~d}_{3 / 2}$ orbital and increasing the atomic number to 58 produces the ground state configuration of cerium. The maximum in the probability remains at nearly the same radius as in lanthanum, but the outer shoulder disappears completely.

It is a matter of interpretation gbout whether the "collapse" occurs between $\mathrm{Ba}$ and $\mathrm{La}$ or between $\mathrm{La}$ and $\mathrm{Ce}$. On the other hand, these results differ from those of Ref. 1 (Figure 8-8 in that work) in that there is a second maximum in the radial probability function. The second maximum is to be expected since the effective potential has a double well but its magnitude will depend on the strength of the potential. 
Figure 2 shows the equivalent set of calculations for the actinide series. An additional maximum occurs near 0.3 Bohr units since the probability is for a $5 f_{5 / 2}$ wavefunction instead of a $4 f_{5 / 2}$ wavefunction. The addition of electrons, while increasing the atomic number, modifies the two peaks at large radius as for the lanthanides. The additional electrons, added first to an s-orbital and then to a d-orbital do not screen the nuclear charge enough to keep the inner well of the double-well potential from deepening. The cuter well remains nearly the same because of the $1 / \mathfrak{r}$ character. The deepening of the inner well pulls the $5 f$ electron in toward the atom, eliminating any appreciable probability of finding the electron outside the so-called centrifugal barrier.

\section{The Thorium-Protactinium Collapse}

The ground state configuration of thorium is one with no $f$ electrons. Instead there are $26 \mathrm{~d}_{3 / 2}$ electrons. As the atomic number is increased by one to give protactinium, the number of $f$ electrons jumps to two. $Z$. Fisk, at Los Alamos, speculated that an analogous collapse should be seen for states with two $f$ electrons in going from thorium to protactinium. Figure 3 verifies this speculation.

Beginning with the cerium-like configuration for thorium shown in Fig. 2, the $6 \mathrm{~d}$ electron was promoted to the $5 \mathrm{f}_{5 / 2}$ orbital. The result is an enhanced probability of finding the $f$ electron at large radius as shown by the shoulder in the radial probability near 20 Bohr units. The atomic number is then increased by one and an additional electron is placed back into the $6 \mathrm{~d}_{5 / 2}$ orbital to give the ground state of protactinium. The shoulder in the $5 f_{5 / 2}$ radial probability disappears. Although this change is not as dramatic as the collapse at the threshold of the lanthanide and actinide series, it is nonetheless present.

\section{Sensitivity to the Exchange-Correlation Model}

The above calculations were all carried out using the Kohn-Sham exchange-correlation potential. The sensitivity of the results to this approximation is tested by adding a correction derived by Gunnarsson and Lundqvist. Figures 4(a), (b), and (c) compare the radial probabilities for the $4 \mathrm{f}_{5 / 2}$ solutions in the lanthanide collapse. Figures 5(a), (b), and (c) show the results for the $5 f_{5 / 2}$ actinide collapse. The dominant trend seen is that the Gunnarsson-Lundqvist approximation enhances the radial probability function at small radius. This feature is less prominent for the highest atomic number in 
each series, but the character of the solutiors for the lowest atomic number is dramatically effected. The qualitative nature of the $f$ electron collapse is still observed in the actinide series, but there is appreciable probability of finding the $5 f_{5 / 2}$ electron at small radius in radiun.

The qualitative picture is significantly altered for the lanthanide series. For barium, the small radius probability is increased to the extent that the collapse is predicted to have already occurred.

This sensitivity to the exchange-correlation model may explain the disagreement between these calculations and those shown in Fig. 8-8 of Ref. 1, but the Gunnarsson-Lundqvist results are not supported by the experimental evidence shown in Fig. $8-9$ in Ref. 1 .

An explanation for this sensitivity may be found in the work of J. H. Wood. 6 His paper points out that the parameterized expression of Gunnarsson and Lundqvist was constructed for a screening radius $\mathbf{r}_{\mathbf{s}} \mathbf{m}$ $(3 / 4 \pi \rho(r))^{1 / 3}>1.0$. The inequality is satisfied for the lanthanides and actinides at radii greater than 1 Bohr unit. The amount of charge contained in a sphere of such a radius is over 95 of of the total. Therefore, most of the charge in these atoms is not properly treated by the Gunnarsson-Lundqvist approximation. This effect was suspected by Wood even for zirconium $(Z=40)$.

This observation should not be considered an inaictment of the Gunnarsson-Lundqvist parameterization (or others like it) but should be only interpreted as a cautionary flag. Wood's results for multiplet splittings show that the approximation is no doubt useful, but if the resulting atomic wavefunctions are to be used to calculate matrix elements, there could be difficulties. For example, the characteristic shift to smaller radius of the probability will obviously effect a dipole matrix element used in calculating photo excitation cross sections.

\section{Additional Sensitivity}

The calculations all have used the Latter tail prescription for the potential. This correction is required to avoid the exponential decay of the atomic potential inherent in the use of a local density approximation to exchange and correlation. Such a decay can exclude having an excited state eigensolution such as the $4 f_{5 / 2}$ state in barium. Imposing a $1 / \mathrm{r}$ behavior on the potential at large radius allows for the presence of these solutions.

The Latter prescription is a popular choice for this type of behavior. However, this procedure does not result as a consequence of a variational principle used to minimize the total energy. A prescription based on the usual 
variational principle would be theoretically more attractive. One such prescription was proposed by D. A. Liberman. 7

The barium calculation shows the largest sensitivity to cise exchange-correlation model potential, so it is also used to explore the sensitively to the tail correction. Figure 6 gives a comparison (using the Kohn-Sham exchange-correlation potential) of the $4 f_{5 / 2}$ radial probabilities for the Liberman and Latter prescriptions. Although the sensitivity to the tail correction is not as striking as that for the exchange-correlation models, it is nonetheless significant.

The qualitative description for the $f$ electron collapse is unchanged. However, the quantitative effect is obvious from the figure. The implications of this effect in the evaluation of matrix elements are also clear.

A more quantitative way to judge the results is to compare computed ionization energies with experiment. The average value of the experimental ionization energy for a barium atom with a $6 \mathrm{~s} 4 \mathrm{f}$ configuration is $0.920 \mathrm{eV}{ }^{8}$ The computed value for a potential using the Latter prescription with the Kohn-Sham model for exchange-correlation interactions is $0.846 \mathrm{eV}$. Using the same model but with the Liberman prescription give $0.664 \mathrm{eV}$. The Latter prescription with the Gunnarsson-Lundqvist model gives an ionization potential of $1.434 \mathrm{eV}$., consistent with a more attractive potential.

The Latter prescription using the Kohn-Sham model gives better results than the Liberman prescription, but the case of barium is probably such a sensitive test that no generalization can be made. However, the barium case can serve as a touchstone for future local density approximations for exchange-correlation and prescriptions for the large radius behavior of the potential.

\section{Acknowledgements}

I would like to thank $Z$. Fisk for raising the query about the $f^{2}$ collapse in going from thorium to protactinium, and A. M. Boring for several discussions about the computational details of the study. 


\section{REFERENCES}

1. Robert D. Cowan, The Theory of Atomic Structure and Spectra, University of California Press, Berkeley, 1981.

2. The code DAVID is a variant of the code HEX, D. A. Liberman, D. T.Cromer, and J. T. Waber, Comp. Phys. Comm. 2 107 (1971).

3. W. Kohn and L. J. Sham, Phys, Rev, 140. Al133 (1965),

4. 0. Gunnarsson and B. I. Lundqvist, Phys. Rev. B13 4274 (1976).

5. R. Latter, Phys. Rev. 99510 (1955).

6. J. H. Wood, J. Phys. B: Atom. Molec. Phys. 13 1 (1980).

7. D. A. Liberman, Thys. Rev. B2 244 (1970).

8. C. E. Moore, Atomic Energy Levels as Derived from the Analysis of Optical Spectra, U. S. Nat. Bur. Stand. Circ 467 Vol. I-III, 1958. 


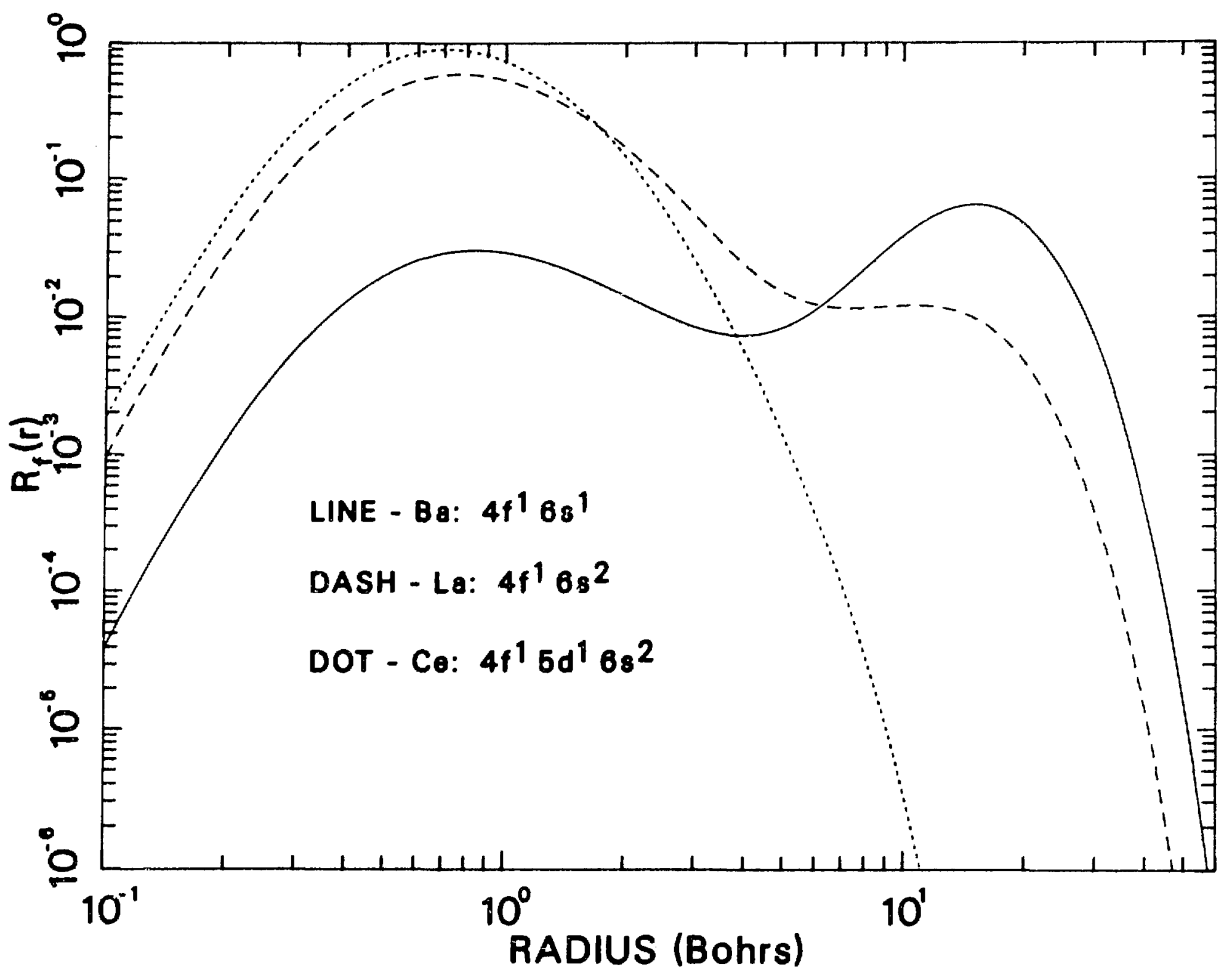

Fig. 1: $4 f_{5 / 2}$ Radial Probability 


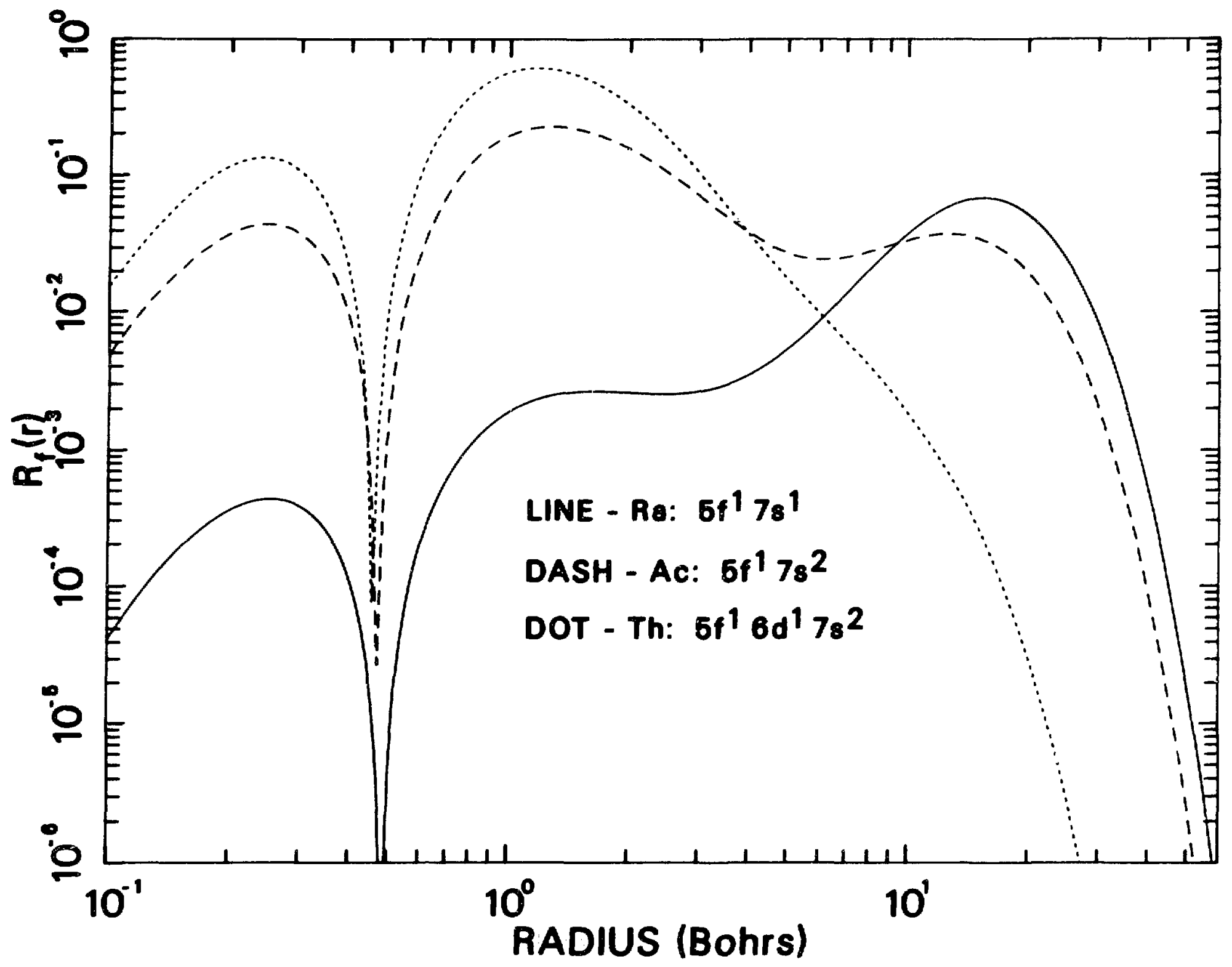

Fig. 2: $5 f_{6 / 2}$ Radial Probability 




Fig. 3: $f^{2}$ Collapse 


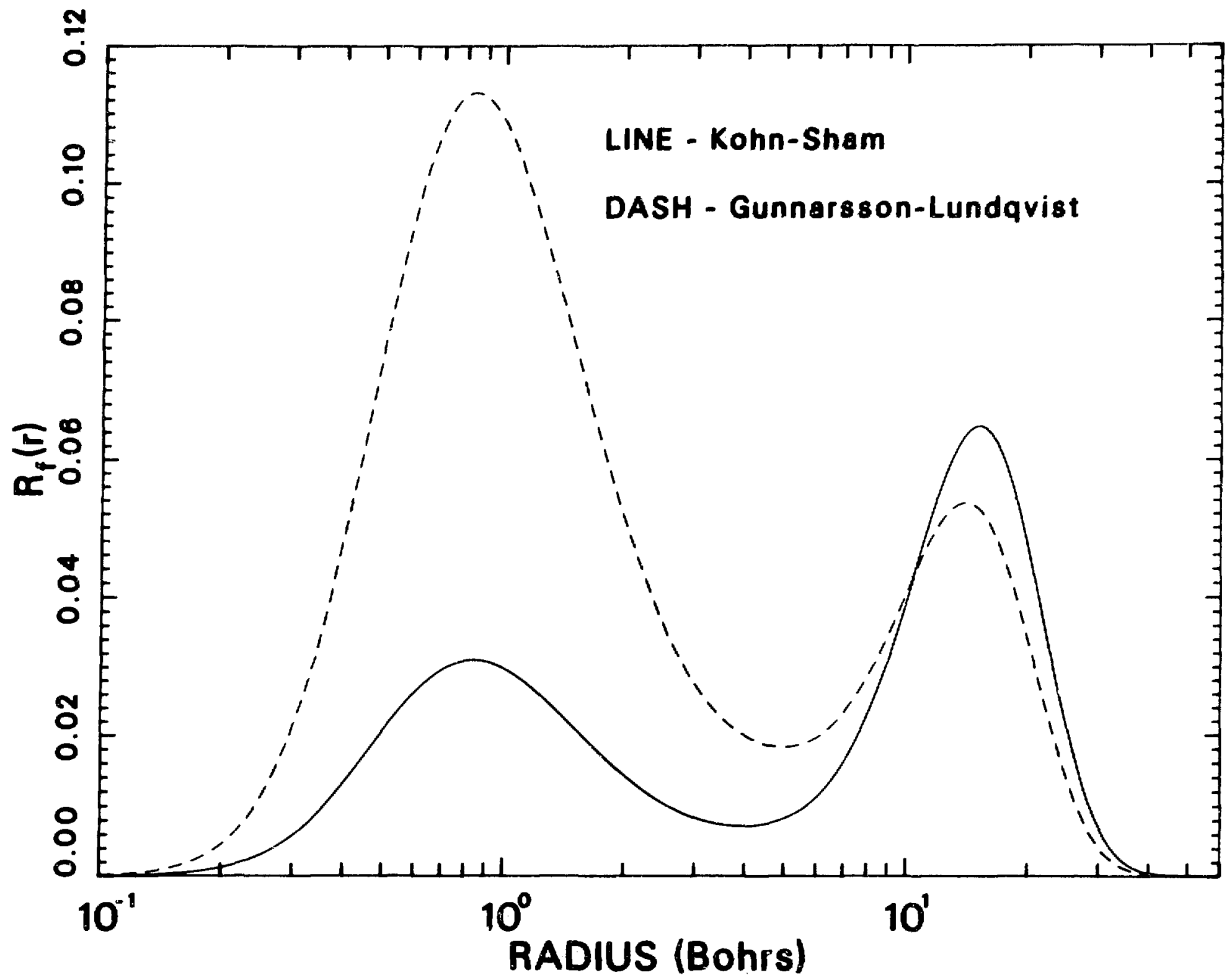

Fig. 4(a): Ba Exchange-Correlation Sensitivity 


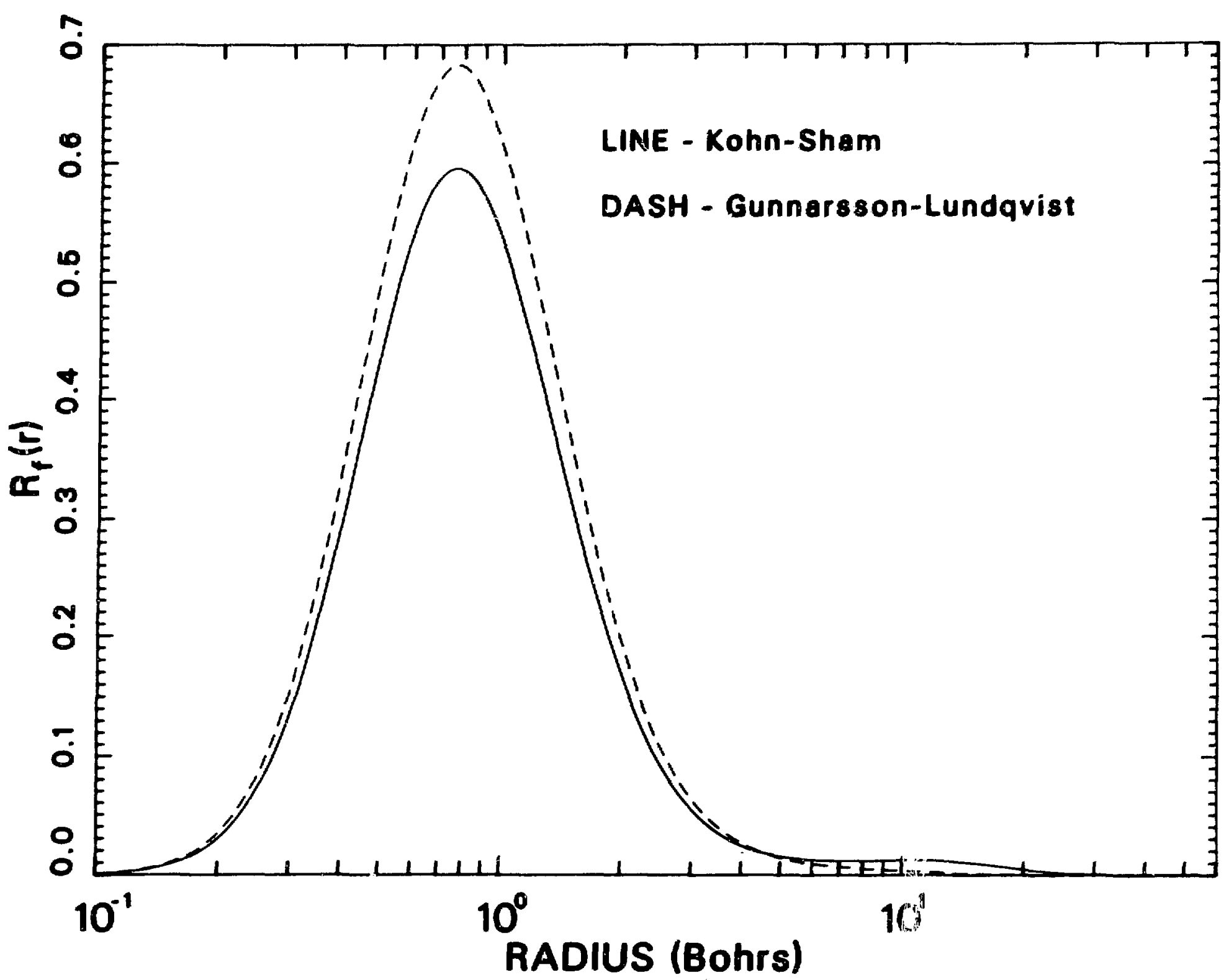

Fig. 4(b): La Exchange-Correlation Sensitivity 


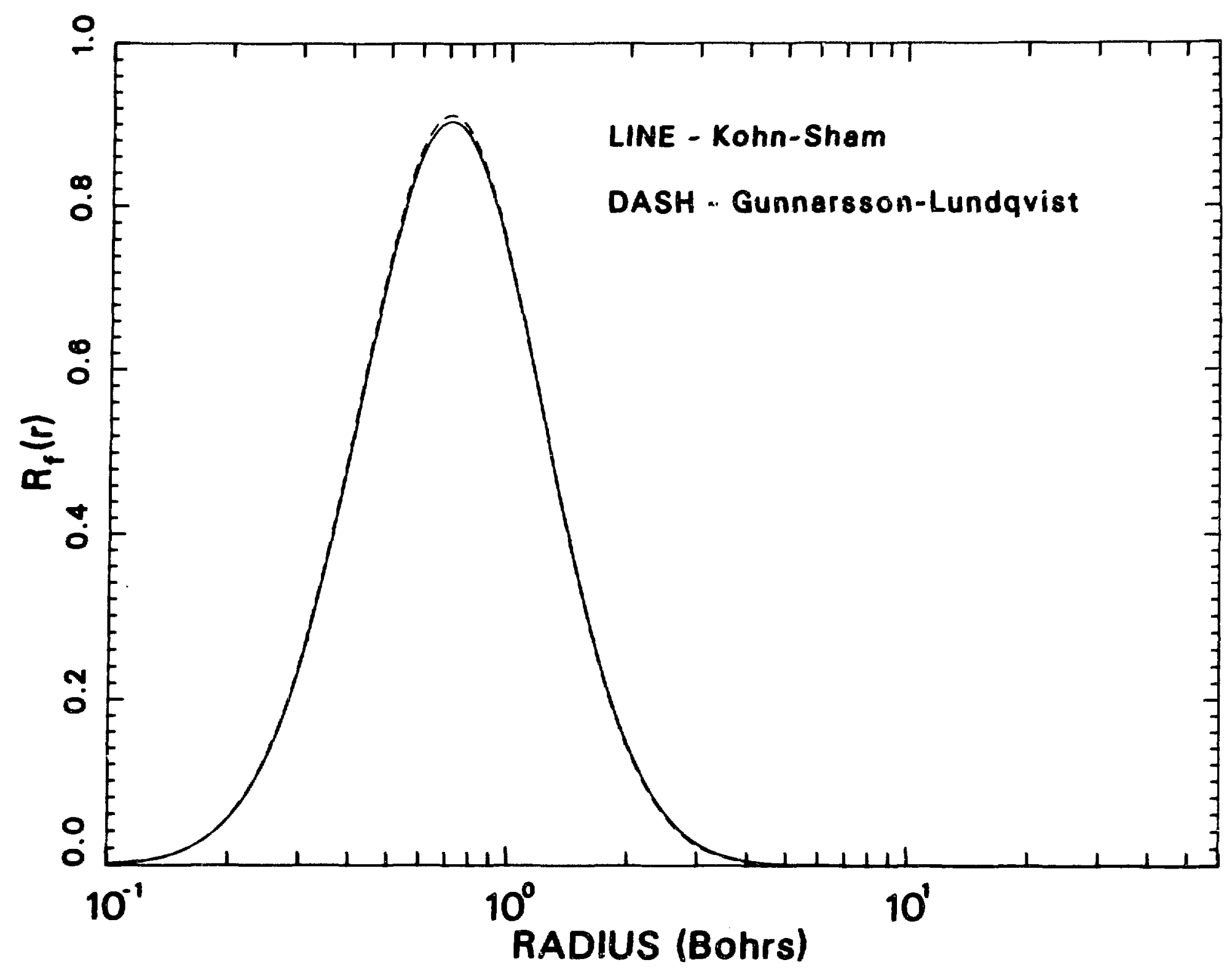

Fig. 4(c): Ce Exchange-Correlation Sensitivity 


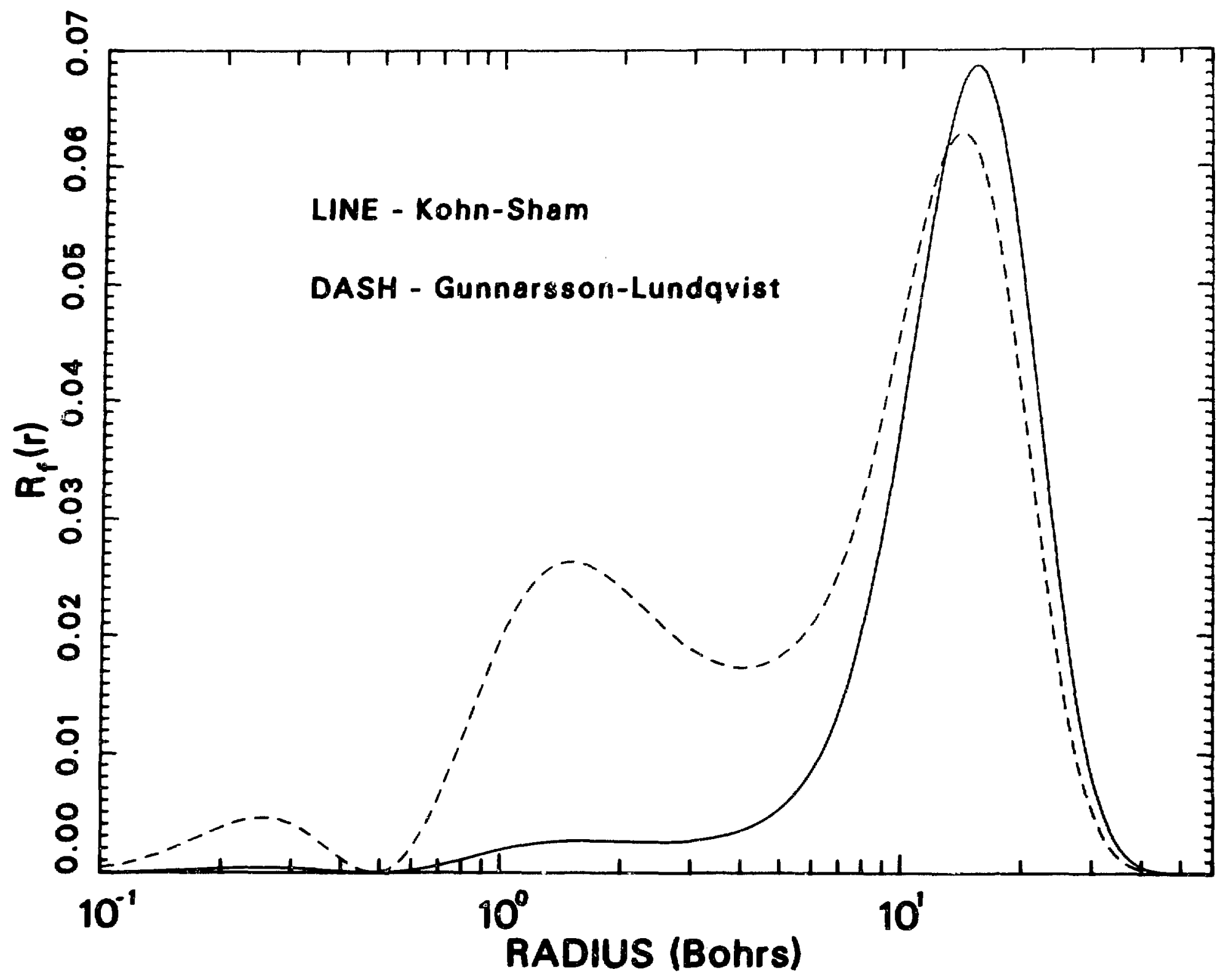

Fig. 5(a): Ra Exchange-Correlation Sensitivity 


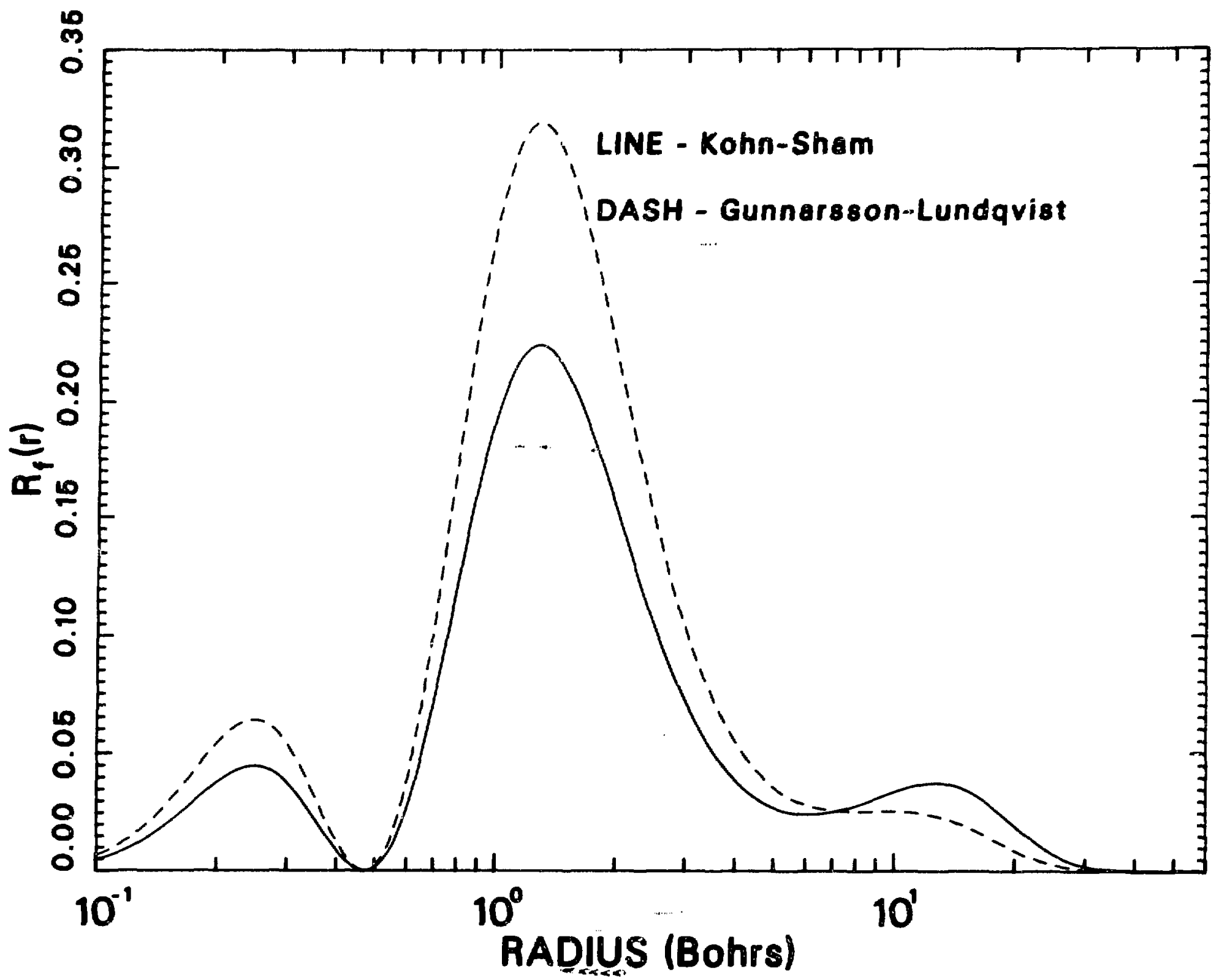

Fig. 5(b): Ac Exchange-Correlation Sensitivity 


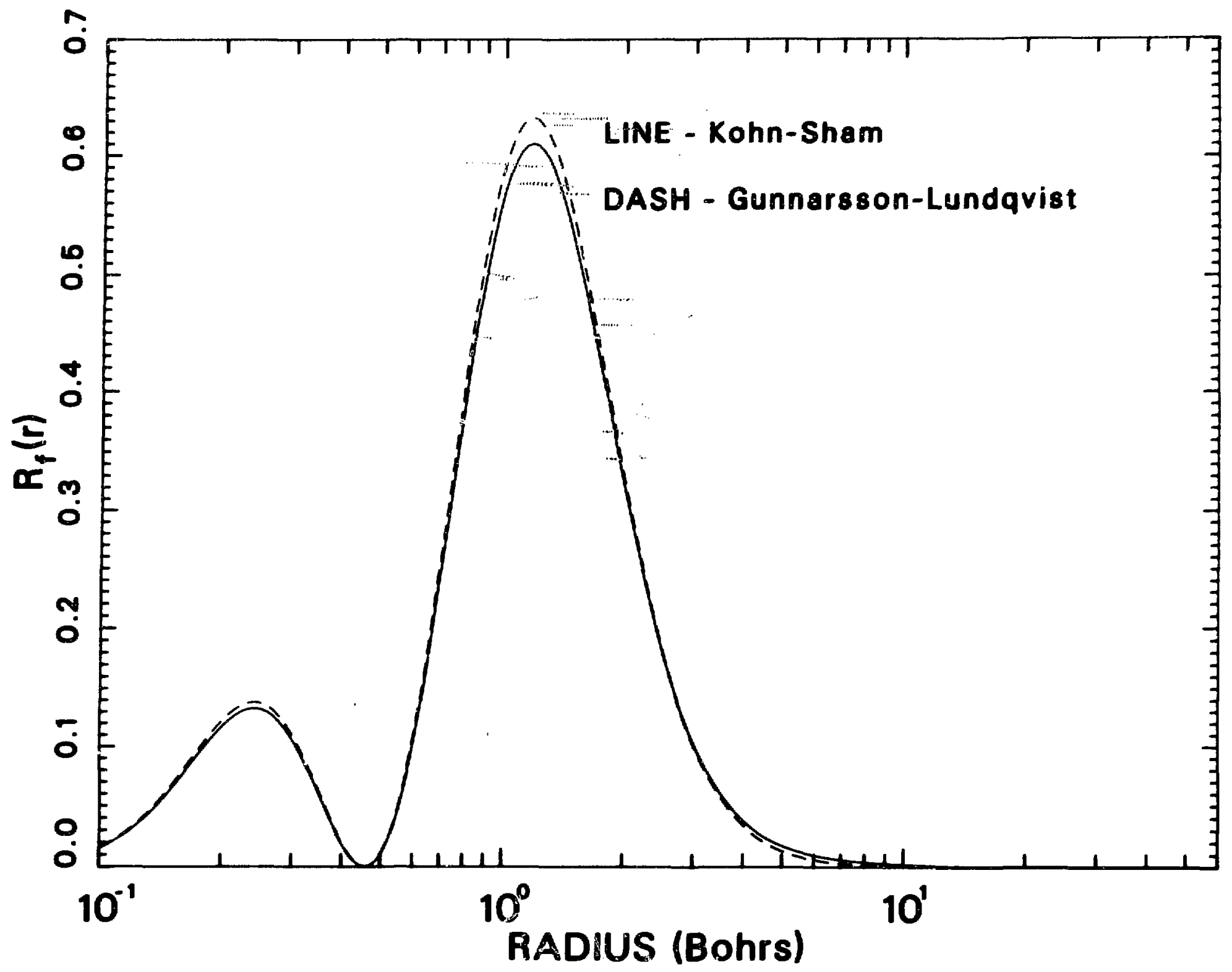

Fig. 5(c): Th Exchange-Correlation Sensitivity 


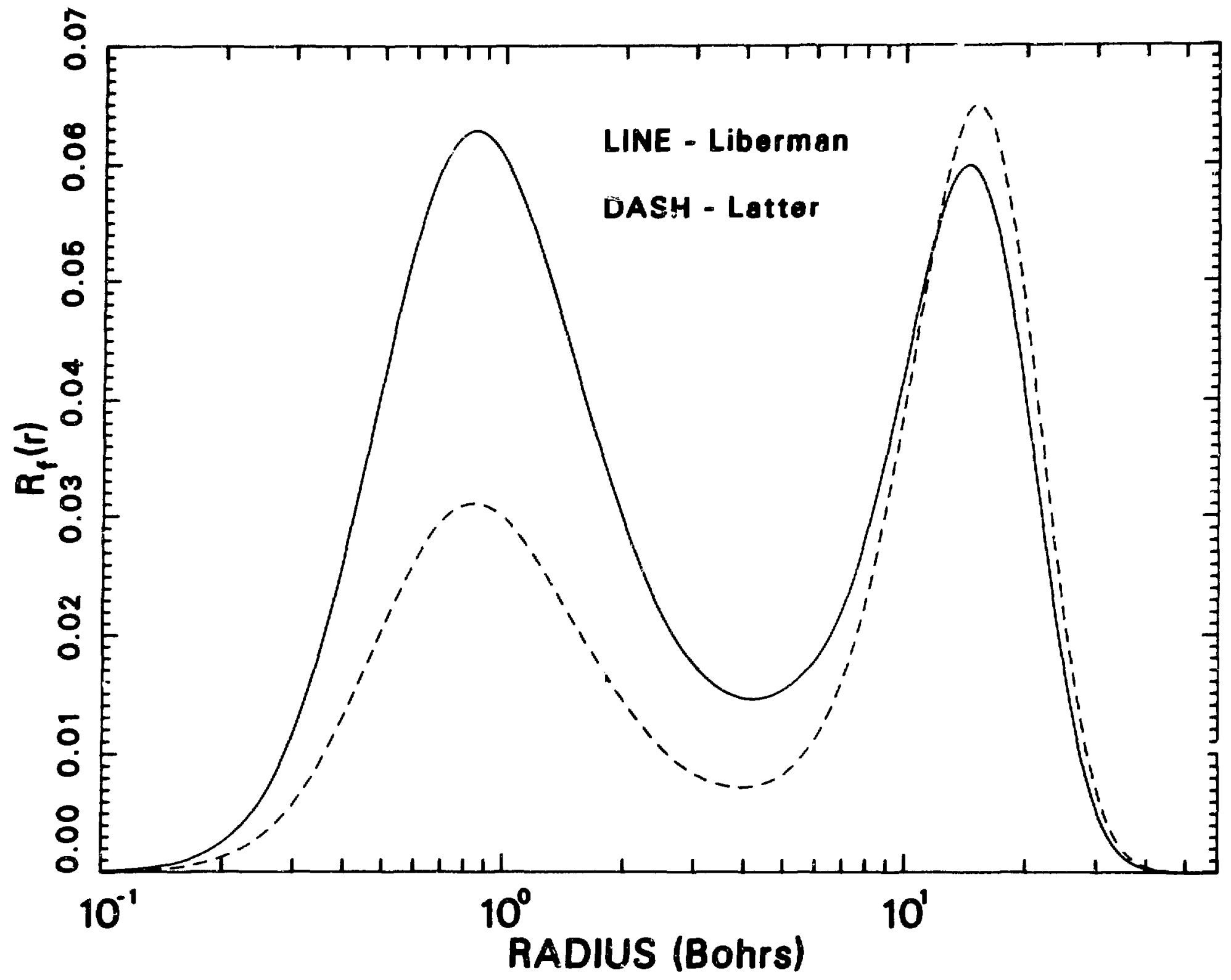

Fig. 6: Ba Tail Correction Sensitivity 